\title{
Universal Science Part I
}

\section{S.D.S. Deshapriya}

\begin{abstract}
This paper is an outcome of a trial carried out to find a common methodology to address the different subjects together rather than treating each separately. It was an effect of, after observing the common features and occurrence among different things. Accordingly the patterns prevailing over different subjects were found. In this respect circular thinking method has been selected as the best way to deal with formation of patterns among different subjects and to address the same. This gave birth to the concept of Universal Science. This paper also describes how Buddhism which comprises of circular thinking (in patterns of thinking), could be used to enhance the Science and other subjects, beyond the limitations of Western Science. Herein, the different subjects are taken together using similarities, patterns and addressing at the elementary level under the Universal Science, rather than treating them separately. Since the subject structure is completed in Buddhism, this unique identity of the Philosophy is used to maximize the various subjects (transformation of Buddhism?).
\end{abstract}

Accordingly the subject of management is also given a broader definition and the subject of law is also raised to a broader logical base, as examples.

This is going to be the future science with valid facts, which covers all the subjects. Delay in the Western Science and getting rid of it by the method of patterns is also explained by taking the ISO as an example.

It could be further shown, that all the methods used for the discoveries in the world have the same methodology which follow for attaining the Nibbana. Accordingly the Five methods are given.

Universal Science gives the most effective way of solving the problems.

Ample benefits which can be achieved through this science is listed out, accordingly. This could be utilized very effectively, when making the Knowledge Hub in the process of heading towards "Ashcharyaya" (Miracle) as well.

\section{Introduction}

Noble objective of the Buddhism is the personal liberation; Buddhism is at the supremacy beyond the material world to achieve this noble objective. It is the complete and fully developed doctrine as far as the general subjects are concerned. According to the Lord Buddha nothing has been hidden as well as nothing to be added or deducted from the doctrine.

Research shows that the potential ability of Buddhism is so vast that it can,not only enlighten the man kind but also enlighten all the subjects in the material world. Buddhism is so strong, either it can redeem the man kind from material world or the entire material world can be shaken \& raised to the climax for the better living of man kind. Hence all the subjects could be improved through Buddhism.
It is possible because of the innovative power, which is inherent in the philosophy.

In this respect, Buddhist philosophy comprises of three interpretations namely the direct meaning, derived meaning and the indirect hidden meaning.

When developing the Universal science it takes basically the above three qualities of the doctrine; (a) Supremacy beyond material world

(b) Completeness

(c) Three interpretations

Eng. S.D.S. Deshapriya C.Eng. MIE(Sri Lanka), B.Sc Eng. (Moratuwa), Senior Engineer, Road Development Authority 
In this context, the concept of US (UNIVERSAL SCIENCE) is developed, so that the different subjects are derived and detailed through Buddhism. Accordingly various subjects are upgraded and expanded by the potential ability of Buddhism, including simulations and the pattern method which is basically the Buddhist thinking pattern. In the light of comprising of three interpretations of the doctrine, it can be stated that Universal Science is entirely initiated through Buddhism.

\section{Objective}

Objective of this paper is to show the potential ability of Buddhism to enhance the other subjects and to withstand as a separate science herein named as "universal science", ahead of material science in many forms. Further showing the possibility of reawakening the new innovative era through our culture. Utilizing of it to form the knowledge hub in the process, towards the Ashcharyaya (miracle) is also another objective.

\section{Methodology}

Under the US, different subjects are taken together utilizing the relationships, rather than taking the subjects one by one separately as in the prevailing science .For this purpose common patterns and the common elementary structures which are found among the different subjects are utilized. When the common patterns \& relationships are found difficult, Buddhism is used to find the same, since it is the complete subject.

(Above all these, the over all methodology used here is basically the similar methods which is described in Buddhism for spiritual gains [new things].)

\section{Interconnections}

Buddhism is based on Chacreya [circular] thinking. This means the repetitive nature of things. Accordingly this repetition is observed among the subjects as well as across the time frame. It is visible when looking at closely. Every 20 years, 50 years occurance of flood and rain in the time frame is one example. Human properties are seen quite similar to the material properties as far as the hardness, toughness, endurance strength etc. are concerned.

This phenomena is classified as the repetition among the different subject fields .Similarly many more similarities can be found. (Some of which are later discussed here.) This reveals the pattern formation in the nature though it is separated into different subjects as it appears. Therefore a lot of similarities, simulating behavior and occurrence could be found among different subjects (in subject's axis) as well as in the time axis. Accordingly, the complexity of the nature is taken into the $(X-Y)$ grid form (as a one preliminary level).

\section{Transformation}

Apparently the subjects are different and we have used to grasp and treat them as separate. Subjects are different as long as we treat them differently. Relaxing the above subjects grasp and grab, would start the mobility and the "subjects free flow "among each other

Great scientist Albert Einstein was able to observe such mobility and a transformation and said that mass is the crystallized form of energy. It is the free flow and transformation between mass and energy. Buddhist thinking pattern shows the mobility and the transformation among everything. It is the extensions thinking and the biggest expansion to the thinking of the Instance. Such Mobility is made possible due to the similarities and the simulations which are occurring among different subjects.

In the global sense it is the transformation of one subject to another. Further, achieving subjects maxima are also possible through Buddhism (by restructuring) due to the fact that it is a fulfilled doctrine already.

This transformation is a unique identity of the philosophy. As stated at the beginning Buddhism is for the soul liberation. (ie) by transforming the Buddhism from its original mode of soul liberation to the subject concerned, it would create a wide range of subject's spectrum at the highest possible level (by rotating $360^{\circ}$ ). As an example, Four Noble Truths would be in the form of illness, cause of it, cure and achieving it under the medical field. Further the four quadrant logic taken from Buddhism is applied in the legal version to maximize the Law field.

This is similar to, changing the radio tune to another channel.

$5 \mathrm{~S}$ concept is also one such similarity, which derived through Buddhism. These are the similarities occurred in a common pattern. If it 
is taken in more general form, all the subject matters are built up as the series of effects of common patterns, along the respective subjects concerned. This would give rise to, how the different subjects are built up.

Any subject development being just only a one track of the above (subjects) spectrum. Accordingly, all the subjects including Law, Education, Health and even politics etc. as well as their relevant institutions are raised to their climax respectively. This is in contrast to the gradual development of subjects in the western science.

Achieving the subject maximum could be better understood by briefing in another form. Accordingly,1)methods adopted to teach the ever, most difficult lesson of "Thrilakshana" would mark the maximum of the education subject.

2) Four quadrant logic in Buddhist foundation would provide the widest base to the present law subject.

Other subjects improvements are also in the same manner. Hence in one way, this shows that reaching the subject maximum through Buddhism is much easier than with the respective subjects alone. This compels to agree that the Buddhism means everything and beyond everything. Simply, it will be more or less a short cut sometimes, for the subject development than attempting right through the individual subjects.

When forming the universal Science, Buddhism plays a vital role mainly in two ways.(a) Finding the inter connections (b) Reaching to the maxima of subjects.

As stated earlier, searching for the Connectivity among different subjects are important. Otherwise unnecessary delays are occurred in the subject developments. For example, ISO concept was delayed in material science due to the failure to see its connectivity to the $3^{\text {rd }} \& 4^{\text {Th }}$ noble truths. This is further explained later under another chapter.

\section{Few Examples}

Hence herein, the connectivity among subjects are further looked into. Accordingly through the patterns, physical models have become feasible for the difficult adhoc situations. Eg: Being the Death always together with birth as shown in Buddhism by a mushroom carrying a bit of soil on top would represent the common pattern of +/- [two opposites] together in the nature, all the time. With this exposure, now the Management subject gets a new face lift, over the traditional definitions. Hence the way of negotiating \& compromising with inherent opposite elements, to achieve the best out of it would be a broader \& more reasonable definition for the management. Further the dealing with two opposites [extremes] would finalize the middle path as the best (compromising) way (Path) of management. Universal science is the grasping of the subjects all together through common patterns and common elementary structure rather than addressing them one by one separately as in pure, Applied sciences.

Other complex type of interconnections is also found among different things. Accordingly, the inter connectivity is found between the material and immaterial world according to the Buddhism .It is a vast expansion to the mass energy transformation. It would give a good opportunity for easy understanding the natural phenomena in immaterial world, physically and in a tangible manner.

For a example, (behavioural) shape of the thinking pattern could be found through this tangible method. Accordingly, it gives that the circular [repetitive] motion of the universe to be the tangible \& physical model of all the nature patterns including the thinking pattern (ie) Resulting the thinking to be in the circular form $\mathrm{V}$-s abstract thinking. On the other hand, it is further verified again, since the nature patterns (of which are repetitive as stated earlier) could be (grasped) understood fully, only by synchronizing the mind [way of thinking] with the same; hence only by circular thinking.

In addition to this, a common pattern is found between the self determination of a nation and a divorce of a couple. This gives rise to the pattern behavior between minute \& massive ones as a magnifying or as the extrapolation. This is very useful to find the behavior of the minute or the massive one when the other one is known

\section{Chemical Changes}

Connectivity among different subjects can be taken to a broader platform even by containing the chemical changes.

Accordingly two different things could be combined on the basis that one is the different 
form of the other under different situation. Eg. piece of paper is in ash form under a different situation of burning.

Everything could be interconnected in this manner. It would give rise to a big boost to the inter subject transformation and the connectivity.

Summarizing the above so far, various patterns between far different entities were found in the basis of., the time axis, among the subjects, between physical \& immaterial world, mind \& external world, between massive \& minute things etc. of which covers everything. Ultimately there are no more separate entities but all are only patterns.

\section{Different concepts}

The concept of Universal science is developed to show the capacity of circular thinking patronized by Buddhism to act as a separate science in place of and ahead of prevailing science. In this context, how the Universal science is structured and empowered by Buddhism is further explained. Commonly two types of connections are found among different subjects in two stages.

(a) At elementary subject structure level

(b) As Simulations at the Subject matter level

Subject matters are interconnected with other subjects through similarities and make the relationships among the different subjects.

Reason for occurring such simulations is because the, elementary structure of all the subjects are the same. Hence it is also taken as an inter connection among the subjects under (a).

All the subjects are still at the developing stage. Hence, situations have arisen that it can hardly find the simulations among subjects or not adequate due to the deficiency in the present (level of) subject structural formations. In order to solve this situation Buddhist philosophy is used in two ways.

(1) To maximize simulations among the subjects.

(2) To make the elementary subject structures [skeleton] perfect. It is possible because Buddhism is already the complete subject.
In other words, by taking the full structure from Buddhism, various subject material [entities] are hung on to it by using cause \& effect links etc., to form different subject bodies .It is similar to the fixing of flesh to the skeleton to form various body shapes. Accordingly, being the Buddhism beyond the material world, any subject in the material world could be revealed through Buddhism up to the maximum level. It is possible because Buddhism is entirely a teaching of cause and effect theory and being beyond the material world. Hence any subject could be reassembled and shown how well it could be contained, totally into Buddhism.

\section{Features of the Universal Science}

Unlike in the prevailing science, connections and patterns which are occurring among the subjects is fully utilized here to get maximum benefits. Main difference between these two sciences is the above factor which would give the great achievement and the advancement to Universal Science. Obviously the entire western science is absorbed into the US and it would get rid of all the ill effects in western science. Therefore in one way Universal Science could be called as the" future science" in place of present Science.

As far as the perfection is concerned, naming this as "universal science" would be quite meaningful due to two main reasons

Taking the quality of (already) completion, as an advantage, Buddhist doctrine is used to upgrade the other developing subjects

$$
\begin{aligned}
& \text { Simulations are found among the } \\
& \text { different subjects hence traditional } \\
& \text { subject boundaries are relaxed and } \\
& \text { theoretically it starts the free flow } \\
& \text { (transformation) across different } \\
& \text { subjects over the boundaries (ie: subject } \\
& \text { liberalization/science globalization) }
\end{aligned}
$$

Since the interconnections among different subjects are formed, it would break the traditional boundaries of prevailing Science. Next era of science would be born at the time of this breakage.

To elaborate further, unique features of this science is given here to compare with the present science. 
a) Since the universal Science is derived from Buddhism (of already completed doctrine) the foundation and the structural formation of different subjects is so broad, strong \& completed from the beginning.

Hence no corrections any denial or deviations would be necessary due to the new findings later on.

b) Being the Buddhism is above the material world subject maximums are obtained by coming down slightly from the (spiritual) concept level (of philosophy).

c) Futher Developments to the subjects are by the downward detailing in contrast to the upward gradual development in prevailing Science.

d) In Addition to this, total subject development in lateral direction as well, through simulations among different subjects (Inter-subjects corporation in lateral direction).

e) Hence the subject development is in extra two directions as well (from top \& laterally at sides) as given in $2,3 \& 4$ in addition to the upward direction, in contrast to the unidirectional upward gradual development in present Science.

f) Hence total subject development is together [integrated] in contrast to the one by one individual development in present Science.

g) Pattern formation is utilized here (for the expedition) against the treating as separate entities in the present Science which is a slow process.

\section{Fast Techniques}

In fact, this is a fast technique for development, by improving the subjects through simulations etc. For example ISO concept could have come to the world long before through this method.

End quality of products came to the world first and ISO concept came after several decades. According to the common patterns and the simulations it could have taken as a simulation from Buddhism promptly.

In the four noble truths, $3^{\text {rd }}$ truth (Nibbana) and the path for it as the $4^{\text {th }}$ truth are the two matters and Nibbana is attained only by following the correct path for it. As far as the material products are concerned above fact could have been simulated as the quality end product and the quality process. Hence ISO concept could have been revealed to the world well ahead to the quality end product or at least together with it.

So, delaying ISO concept is an one incident only. It is in general the born defect of this way of thinking i.e. treating separately one by one without searching for the relationships among different things.

In general, prevailing science is constantly suffering from this main decease of delay.

In fact this thinking pattern is responsible for delaying the scientific development over centuries. Cumulative delay occurred so far is so long. In the light of occurring the pattern formation among the subjects and the nature in general, the main defect in the abstract thinking and hence in the prevailing science, is the neglecting of this fact. [Ie. the Patterns].

Hence to correct the situation, pattern formation should be given the priority before treating the subjects separately. It is the only way to correct this defect.

Therefore the concept of universal science is there to take up the challenge of eliminating said delaying by introducing fast techniques.

\section{Discoveries \& New Innovations}

Finding the path to Nibbana is a new innovation and a new revealing to the world after billions of years by the Lord Buddhas from time to time. Hence, Innovative power of Buddhism is so strong that all the new inventions \& discoveries has been carried out by the scientists in the world only through the methods follow towards Nibbana.

All the innovations in the world have been done by following similar procedure as attaining the Nibbana. No invention has been carried out in the world by any other means.

According to Buddhism, following the eight noble path is the only way to Nibbana. While following the noble path, it can be observed that there are few techniques \& methods to be applied for achieving this noble objective [Nibbana] depending on the Personal intelligence \& the situation etc.

These methods \& techniques are basically classified into five groups. 
(1) Complete set of four noble truths.[complete process of innovation]

(2) Cause \& effect method (diminishing the cause \& changing its effect.).

(3) First Amulika Sradda followed by the Akarawatte sradda.

Assuming the solution \& reaching for it there after (Hypothetical method).

(4) Approaching thrilakkana pattern through different accesses (Such as decaying skeleton, descending flower, diminishing oil lamp etc) [simulating \& patterns method]

(5) By correct practical approach of emphasizing the $3^{\text {rd }}$ truth \& followed by the $4^{\text {th }}$ truth. [correct orderly process]

Since these are the only methods for innovations \& creativity, this will become the only culture in the world which promotes creativity as an its inherent feature. Our past glorious history gives evidence for the same.. Secrecy behind our glorious past is the innovative culture that we had practiced .At the time we were able to become the pioneers of steel manufacturing. Universal Science is developed here again for the resumption of the "Innovative Era" through the culture ,from the point where we had stopped after the steel manufacturing first time in the world history .Hence this will be the beginning of the cultural revolution for new innovations.

\subsection{Method of four noble truths:}

Four noble path is the entire process of attaining Nibbana starting from the point of problem definition (Dukka).Before searching for a redeem, it is the prerequisite or necessity to realize the problem of Dukka.

Lord Buddha has categorized and defined the Dukka very elaborately as the 1st truth at the beginning. Before searching for a redeem it is a prerequisite of problem definition.

It is important to understand the ever lasting bond of Dukka, which the freedom is sought from Then only the cause of Dukka, solution (redeem) and path to solution is found. As far as the complete process is concerned starting from the problem definition is important.
These steps are the full set of procedure for new innovations. As an example this procedure is applied to the user friendly new products generation. Problems faced by the user has to be correctly assessed and identified by the inventor before upgrading it as a new product. This is how the user friendly next generation of cars, computers etc. are invented.

It is a continuous process of research and survey starting from need upto the upgrading level (new generation) Further during the process of inventing new products, usually some problems are arised interrupting the new invention. Then the inventor checks for it and identifies the problem, followed by finding out the cause of it. Thereafter the solution is found by the inventor by eliminating the course. During his process final stage is the action taken to make it a reality. Before any invention these are the four stages to be carried out in the process.

\subsection{Cause and effect method:}

Note:

\#\# any thing can be changed by changing the causes for it.

\#\# Changing the causes would change the effect.

This procedure could be easily explained by considering the invention of new chemical products.

In a chemical laboratory new products are invented by mixing and extracting the variety of chemicals. In this process it would diminish the causes, which prevent the required quality, until it reaches the final desirable quality.

Final desirable effect (Product) is reached by diminishing the unwanted reasons (causes)behind, and promoting positive causes (+ ve values )This is the similar procedure for attaining Nibbana by diminishing the causes for sansara (Thrushna) and by the way of adding merits. (+ ve valves).

\subsection{Hypothetical Method (Sradda Approach)}

Before making any new product initial concept is drawn in the mind. There after the detail analysis is done for practicing (to make it a reality) This is a fundamental method behind any innovation. This is so important that 
without this technique no innovations are possible.

In mathematics also, this technique is used to solve some difficult questions. Making a Hypothesis and proving it thereafter is the procedure followed therein

It is the same method followed by the traditional Buddhists for Nibbana through the Sradda approach, (ie). the Amulika Sraddha (concept) followed by Akarawathe Sraddha (Realizing and Reaching gradually) is the method for the Nibbana, which follows.

\subsection{Common Patterns and Simulating Method}

Meditater normally uses some reference objects for realizing the Trilakshana pattern.

Decaying skeleton, withering flower, diminishing lamp are some of the objects using there in. This means the unique pattern of Trilakshana which comprises of impermanency appeares in different forms and outlook over the subjects i.e. same Trilakshana pattern appears in different forms.

\# Accordingly, Meditater enters (realizes) to the common Thrilakshana pattern through any object as above.

\# In a similar manner, Sir Isaac Newton entered the common gravitation pattern (field) through a fallen apple.

\# On the other hand, withering flower, would be appearing as a diminishing oil lamp in another form while in the common Thrilakshana pattern.

\# Similarly, shape of the Benzeen structure appears as in (rounded) snake form when entered into a common pattern while dreaming (through a dream )

In general, this gives rise to the two innovative school of thoughts (a) common patterns, connections or similarities are prevailing over different things.

(b) Same thing appears in different forms and shapes over different subjects. This pattern thinking is very much used for the discoveries in different subjects.
Further the transformation between energy and the mass as discovered by Albert Einstein could be re-expressed in this school of thought as the same thing in different forms (similarities).

\subsection{Innovations by correct orderly procedure}

When attaining Nibbana correct order is the $3^{\text {rd }}$ truth followed by the $4^{\text {th }}$ truth.

(3 $3^{\text {rd }}$ truth $\rightarrow 4^{\text {th }}$ truth) $\sqrt{ }$

not in the other way around.(4th truth $\longrightarrow 3^{\text {rd }}$ truth) $X$

Solution is followed by the path to the solution. Once the solution is decided, practical way of making it a reality is a different issue (as next step) to be attended separately. ie: the action plan has to be worked out to make it practical. Solution has to be finalized first correctly to reach it, by the action plan.

According to this order (of $3 \rightarrow 4$ ), solution is predominant over the path for it and its practicality.

If the practicality is concerned, prince Siddhartha would never reach beyond Newasanna Nasannayathanaya $\left(8^{\text {th }}\right.$ immaterial state) because beyond that, no other spiritual level was practical at the time.

Similarly if mere practicality is given the priority, no new product or solution will be created for ever. It will be the ever stagnant situation which prevails all the time without any progress and never struggle for new things. As far as the effort taken by the scientists is concerned, to discover new products and reach the target, importance of this practical order could be realized.

(In this respect, it would be quite obvious that the many new things becoming a reality had been barred not due to the natural reasons or the lack of technology but by the man made barriers, influences, limitations in law provisions etc. of which could be broken easily at no cost or without any new technology.)

\section{Applications}

Further some of the feasible and interesting achievements which could be gained locally as well as globally are highlighted here as an eye opener to the universal science.

(1) Prevailing law system could be expedited \& expanded with new dimensions. 
(2) Present education system could be improved \& the problems from over weighted school bag to the struggle for popular schools etc. could be addressed by improving the method of teaching.

(3) Problems in the health sector could be addressed successfully, cost for the drugs could be reduced drastically \& the more healthy society could be formed at a lesser cost.

(4) Innovations \& discoveries are promoted since it becomes a part \& parcel of the culture and followings are some of the benefits to the society.

(a) children shall become more innovative; inventing societies in schools and Dhamma Schools are formed.

(b) Field of Art could be raised to the innovative solutions level from the retaining [situation of] trapped and stagnant problem level. (Art of solutions)

(c) Conceptual solutions are formed initially so that the time for trial \& error method could be eliminated.

(d) Hence the cost of experiments could be reduced.

(e) From conceptual level, to detailed solutions at the final stage, could be found

Hence total solutions are found

(f) Solutions are found for long lasting problems

(g) Positive thinking improves and attitudes are also changed accordingly.

(h) Entire social structure could be given a face lift with innovative mode

(i) Development could be expedited

(j) Society becomes problem free hence relax, calm and more innovative

(k) Field of appropriate technology improves

(l) more innovative, cost effective solutions in place of traditional costly methods

(m) New innovative era begins in every field

(All the subject fields are improved) (n) Innovative and alternative methods could be found

(5) Problems could be prevented beforehand by foreseeing the situations

(Preventive actions rather than waiting till curative stage )

(6) Dialogs could be end up this unanimous decisions and conclusions

(7) In the global sense, trouble free balance development is achieved and prevailing problems solve

Hence, Improve the humanity and world peace

(8) Gradual and steady development is reached and sudden draw backs and collapses eliminated.

Patterns and simulations play a important role when finding solutions to

Problems which cannot be solved by abstract thinking, could be solved by collective (curve) thinking.

When cause and effect methods fail, solutions are found by simulating methods.

(a) Be successful when answering the unsolved questions in intangible fields such as universe, mind etc

(b) When no solutions are found through the subjects directly, this acts as an alternative methods for solutions.

(c) In addition to this, Costly survey and research could be avoided

(d) Upgrading the weak subjects by simulations

(e) Forecasting, predictions and extrapolation improves

In brief, Sri Lanka being in the process of marching for the miracle (Ashcharyaya), it is no lesser thing than the Universal Science would have to be utilized, to make the "Knowledge Hub" of the world. 


\section{Solutions to the Problems}

Further with regard to the patterns technique, solutions could be found easily rather than treating and trying from the beginning as a new problem.

\section{Solutions}

* From the (Buddhist) doctrine since it is the only fulfilled subject.

* $\quad$ From the past period, including Jataka stories, since similar things have occurred repeatedly in the time frame (as past experience)

* $\quad$ From other subjects since same thing appear in different form among the subjects

* From other countries since the same thing may have occurred earlier elsewhere in the world.

No problems are erupted without the solutions. Every problem is having a solution. Problems are not new and may have already arisen elsewhere. If the solutions are not found so far, reason for the unsolved situation is simply due to treating and trying for solutions as "NEW" problems. It is an error in thinking, which lacks circular thinking .

Example given here is to, further show the gravity of pattern thinking. The recently occurred Tsunami killed several thousands of people as an first experience of ours. However the effect of the Tsunami could have been minimized since it happened to be nearly the $4^{\text {th }}$ repetition in the recorded history, being its $1^{\text {st }}$ occurrence in Samudra vane jataka.

Other reason for the problems being the selfishness; Most of the problems are due to the selfishness; It is once again due to the direct result of lack of circular thinking which promotes group feeling.

By taking categorically, living with the problems, unsolved, seems to have become a part of our culture and the corrective action is not taken to solve them. Hence it is mainly a cultural problem. In this context, best results could be obtained by a cultural approach, rather than addressing the problems one by one separately. Cultural approach which comprise of circular thinking will solve the problems promptly. That is how, during the past, our ancestors have solved the problems and preventing them successfully, by taking as a part and parcel of the culture. Through the Universal Science, would be the best approach for solving the problems as in the past. Accordingly, the preventing and eradication of the problems would take a main part of the Cultural Revolution as mentioned earlier, which is heading towards the new innovative era.

As stated earlier, almost all the problems including environmental issues such as global warming occurs as a result of the struggle for individual benefits among powerful countries. This is purely due to selfishness. According to the circular thinking pattern, it is revealed that the selfishness is a direct result of present way of thinking. All these crises are due to the error in the thinking pattern.

Nature itself is a pattern and it comprises of patterns. In broader aspect, all the problems are arisen as a result of grasping the pattern as individual and separate entities, which ultimately ends up with individualism or selfishness (separatism)

As said earlier it is a born defect of abstract thinking which lacks the patterns and group thinking.

In the circular thinking it promotes patterns and inter-Subjects Corporation through simulations and similarities. This build up the group feeling friendship- and the interconnectivity .Hence the pattern thinking would create a friendly environment which improves the humanity. Changing to the pattern thinking would start a revolution for the better world by eliminating the world problems which were created due to selfishness. It will be a cultural revolution at the global scale.

It would end up with resulting the everlasting world peace with the balance development in every aspect. In a broader sense, Universal Science which combines with pattern thinking would be the new world order and create a new world for the man kind and the future generation. 


\section{Conclusions}

This study investigated the pattern behavior which was found in various things. Buddhism is utilized here to further enhance the above property and the reasons behind for such enhancing ability are mainly the circular thinking pattern, (completed) structural formation etc. of Buddhism. Examples given here shows the logical relationship among the difference things. Ample benefits which can be obtain through circular thinking shows its affect for fast development. In this connection specially the pattern method, which can be used as a fast techniques is illustrated here taking the ISO concept as an example. Existence of various patterns are also addressed in very broader scale by circular thinking. In addition to this, as a cultural approach, new renovations can be boosted, since similar methods and techniques were followed to attain Nibbana, as explained here. Hence new innovative era can be started from Schools and Dhamma school level. Further the New knowledge can also be gained fast accordingly in various subjects in conceptual level as well for further subject developments. Hence this could effectively be used to form the knowledge hub.

\section{References}

1. News Paper articles including "Mihindu himiyange Amba Prashnaya" of Professor Nalin de Silva

2. News Paper articles of Buddhist Scholar, Professor Hettiarachchi and others. 